\title{
Crystallographic Characterization of Polycrystalline Materials: High Resolution Automated Crystallite Orientation \& Phase Mapping and Precession Electron Diffraction Ring Patterns
}

\author{
S. Rouvimov, ${ }^{*}$ P. Moeck, ${ }^{*}$ E.F. Rauch, ${ }^{* *}$ Y. Maniette, ${ }^{* * *}$ and D. Bultreys*** \\ * Nano-Crystallography Group, Department of Physics, Portland State University, Portland, OR \\ 97207-0751 \\ ** SIMAP/GPM2 Laboratory, CNRS-Grenoble INP, BP 46101 rue de la Physique, 38402 Saint \\ Martin d'Hères, France \\ *** NanoMEGAS SPRL, Boulevard Edmond Machterns No 79, Saint Jean Molenbeek, \\ Brussels, B-1080, Belgium
}

The electron backscatter diffraction (EBSD) technique (also known as orientation imaging microscopy or the back-scatter Kikuchi diffraction method) in a scanning electron microscope (SEM) is often employed for structural characterization of thin polycrystalline films and ensembles of nanocrystals in the powder form. This technique is, however, sensitive to the plastic deformation state of the crystals as well as to structural damage or contamination of the crystal surfaces. In addition, its spatial resolution is limited to somewhere between 20 to $80 \mathrm{~nm}$. Parallel illumination electron diffraction in the nano-probe mode in a transmission electron microscope (TEM) that is equipped with a field emission gun, on the other hand, delivers a significantly higher spatial resolution as compared to EBSD in a SEM and is also less sensitive to the plastic deformation state and the surface of nanocrystals. An automated technique for the mapping of crystallite phases and orientations of polycrystalline materials in a TEM has, therefore, been developed recently [1]. This technique is based on template matching of experimental electron diffraction spot patterns to their pre-calculated theoretical counterparts. Very promising results have so far been obtained with this technique for polycrystalline metal films, microelectronic composite structures (Fig. 1), and inorganic nanocrystalline powders [15]. The procedure of crystallite orientation and phase mapping comprises the automated collection of single crystal precession electron diffraction patterns on an external digital camera while scanning the area of interest with a nanometer-sized primary electron beam, followed by off-line data processing. Spatial resolutions of a few nanometers can be obtained on a fieldemission TEM. Figure 1 shows a typical crystallite orientation map (courtesy of JEOL, Tokyo). The software that goes with this hardware is flexible in its intake of experimental data so that it can also create crystallite orientation and phase maps of nanocrystal from the amplitude part of Fourier transforms of high resolution TEM images [4,5]. For inorganic nanocrystals with small to medium sized unit cells, an objective-lens aberration corrected TEM needs to be utilized [5]. It has also been demonstrated that the single crystal precession electron diffraction (PED) mode [6,7] improves the reliability of this technique significantly as the $180^{\circ}$ ambiguity in the indexing of spot patterns from the zero order Laue zone can be reliably overcome $[2,4]$. This is because more reflections are excited in PED patterns and there are frequently also reflections from higher order Laue zones.

For inorganic nanocrystal without heavy elements and sizes of below some 10 to $50 \mathrm{~nm}$, the intensities of PED reflections are quasi-kinematical (i.e. roughly proportional to the square of the modulus of the structure factors) [6,7]. Such PED patterns are, therefore, very useful for advanced structural fingerprinting of nanocrystals in a TEM $[5,8,9]$. 
"Powder PED" on ensembles of nanocrystals is also possible with a precession electron diffraction add-on to older and newer midvoltage TEMs from the NanoMEGAS company. The electron diffraction rings of the resulting PED patterns are typically more numerous and uniform and the effects of textures on these rings are suppressed [5], especially when large precession angles are utilized, Fig. 2. The intensity of the electron diffraction rings of powder PED patterns is also closer to the predictions of the kinematical theory [10].

\section{References}

[1] E.F. Rauch et al., Microsc. Analysis, Issue 93, November 2008, S5.

[2] E.F. Rauch et al., Special Issue on "Precession Electron Crystallography" of Zeits. Krist. 225, issue 2-3, 2010, in press.

[3] S. Rouvimov et al., Proc. 2009 NSTI, Vol. I (2009) 421, ISBN: 978-1-4398-1782-7.

[4] P. Moeck et al., Special Issue on "Interfacing Methodologies, Device Concepts and Architectural Strategies for Nanoscale Sensors and Systems" of the IEEE Transactions on Nanotechnology, in press.

[5] P. Moeck et al., In: Electron Crystallography for Materials Research and Quantitative Characterization of Nanostructured Materials, Mater. Res. Soc. Symp. Proc. 1184 (2009) 49, ISBN: 978-1-60511-157-5.

[6] R. Vincent and P. Midgley, Ultramicroscopy 53 (1994) 271.

[7] P. Moeck and S. Rouvimov, In: Drug Delivery Nanoparticles: Formulation and Characterization, Informa, New York, 2009, pp. 270-313.

[8] P. Moeck and P. Fraundorf, Zeits. Krist. 222 (2007) 634; arXiv:0706.2021.

[9] P. Moeck and S. Rouvimov, Special Issue on "Precession Electron Crystallography" of Zeits. Krist. 225, issue 2-3, 2010, in press.

[10] This research was supported by grants from Portland State University's Venture Fund, the Army Research Laboratories, and the Oregon Nanoscience and Microtechnologies Institute (ONAMI). Prof. Peter Moeck's “Laboratory for Structural Fingerprinting and Electron Crystallography" at ONAMI serves as the first demonstration site of the NanoMEGAS company in the Americas. The automated crystallite orientation and phase mapping system "ASTAR" which works on the basis of either a second generation (digital) "DigiStar" precession electron diffraction device or a first generation (analog) "Spinning Star (P30)" device can be demonstrated there on request (to pmoeck@pdx.edu or tel.: 503725 4227).

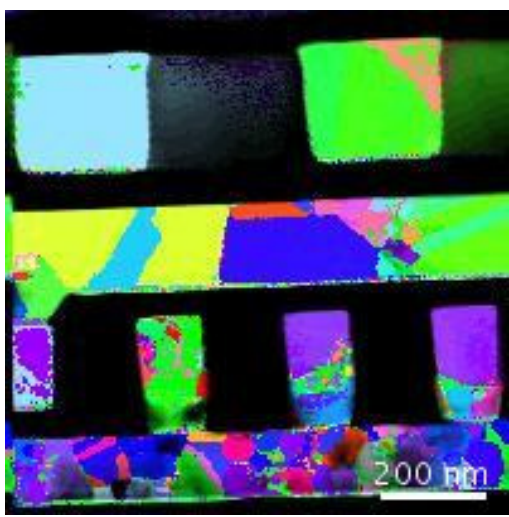

FIG. 1. Crystallite orientation map of $\mathrm{Cu}$ in a semiconductor device (JEOL JEM-2100F).
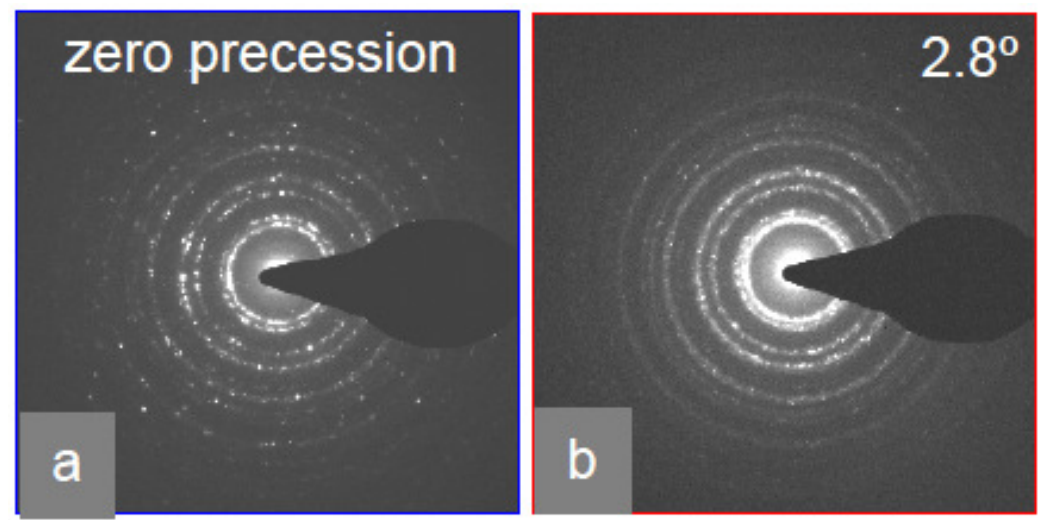

FIG. 2. Effects of the precession angle on the powder electron diffraction pattern of the same ensemble of $\mathrm{Au}$ nanocrystals; (a) $0^{\circ}$ and (b) $2.8^{\circ}$ (FEI Tecnai G ${ }^{2}$ F20 ST). 